\title{
Estimativas volumétricas para plano de manejo florestal na Amazônia Oriental
}

Este estudo teve como objetivo ajustar modelos matemáticos para determinação de estimativa volumétrica para todas as espécies comerciais para área de manejo florestal no município de Placa, estado do Pará, Brasil. Para o ajuste foram selecionadas 235 árvores, distribuídas entre 14 das 17 espécies de valor comercial autorizadas para corte na Unidade de Produção Anual (UPA). Estas foram selecionadas a partir de uma Amostragem Aleatória Simples (AAS), com todo rigor, para que todas as classes diamétricas a partir de $50 \mathrm{~cm}$ e com amplitude de $10 \mathrm{~cm}$ fossem representadas. As avaliações das 6 equações tiveram resultados satisfatórios, sendo que com os parâmetros analisados qualquer uma poderia ser utilizada na estimativa volumétrica para o segundo Plano de Operação Anual (POA). Isso, porque ambas obtiverem baixo erro de estimativa e bons $\mathrm{R}^{2}$ ajust, boa dispersão de resíduos, além de volumes estimados equivalente ao da cubagem. A precisão dessas equações foi sustentada pela alta correlação do DAP $(r=0,95)$ em detrimento a $\mathrm{Hc}(r=-0,23)$. A Hc quando obtida com boa precisão pode gerar equações mais eficazes para estimativas de volume, contudo, se não tiver boa correlação poderá ser inócua. Por isso, deve se ter cautela ao utilizar as variáveis independentes e considerar a correlação antes de testar os modelos.

Palavras-chave: Equações; Variáveis Independentes; DAP; Hc; Floresta Nativa.

\section{Volumetric estimates for forest management plan in the Eastern Amazon}

This study aimed to adjust mathematical models to determine volumetric estimation for all commercial species for forest management in the municipality of Placa, state of Pará, Brazil. For the adjustment, 235 trees were selected, distributed among 14 of the 17 species of commercial value authorized for cutting at the Annual Production Unit (APU). These were selected from a Simple Random Sampling (SRS), with all rigor, so that all diametric classes from $50 \mathrm{~cm}$ and with an amplitude of $10 \mathrm{~cm}$ were represented. The evaluation of the 6 equations had satisfactory results, and with the parameters analyzed any one could be used in the volumetric estimate for the second Annual Operation Plan (AOP). This is because both have low estimation errors and good $\mathrm{R}^{2}$ adjustments, good dispersion of residues, in addition to estimated volumes equivalent to that of cubing. The accuracy of these equations was supported by the high correlation of DAP ( $r=0.95)$ to the detriment of $\mathrm{Hc}(r=-0.23)$. Hc when obtained with good precision can generate more efficient equations for volume estimates, however, if it does not have a good correlation, it can be harmless. Therefore, care should be taken when using the independent variables and considering the correlation before testing the models.

Keywords: Equations; Independent Variables; DAP; Hc; Native Forest.

Topic: Uso de Recursos Naturais

Reviewed anonymously in the process of blind peer.
Received: 02/12/2020

Approved: $21 / 02 / 2021$

Diego Ribeiro de Aguiar

Instituto Nacional de Pesquisas da Amazônia, Brasil

http://lattes.cnpq.br/9241060544837430

http://orcid.org/0000-0003-4013-8664

conctribeiro@gmail.com

Marcos Leandro Garcia (D)

Universidade do Estado do Mato Grosso, Brasil

http://lattes.cnpq.br/2383889571304731

http://orcid.org/0000-0001-9174-4803

ml.marcos@unemat.br

Referencing this:

AGUIAR, D. R.; GARCIA, M. L.. Estimativas volumétricas para plano de manejo florestal na Amazônia Oriental. Nature and Conservation, v.14, n.1, p.149-157, 2021. DOI: http://doi.org/10.6008/CBPC23182881.2021.001.0017 


\section{INTRODUÇÃO}

As constantes mudanças no uso da terra na região Amazônica comprometem a utilização dos recursos madeireiros, os quais são de grande demanda tanto no mercado nacional quanto internacional. Existe uma necessidade de otimizar os processos de estimativas volumétricas de madeira para auxiliar no planejamento do manejo de florestas tropicais, devido à grande necessidade de abastecimento e para a conservação dos seus recursos. A grande extensão da floresta pode garantir um suporte para explorações futuras desde que atendam os critérios de sustentabilidades baseados nos princípios ecológicos, econômicos e sociais.

A demanda de madeira como matéria-prima para a indústria, construção e fontes de energia está aumentando diariamente devido ao crescimento populacional e o desenvolvimento econômico (ETIGALE et al., 2014). Dessa forma, a sustentabilidade do uso dos recursos madeireiros disponíveis e seus benefícios requerem um planejamento adequado, que por sua vez requer o conhecimento da extensão do estoque em crescimento na floresta (AKINDELE et al., 2001). Nesse contexto, a estimativa de volume do povoamento é importante para a tomada de decisões e gestão eficiente dos recursos florestais. As estimativas de volume do fuste são úteis no inventário florestal, porque o volume de madeira é a unidade de gestão básica das florestas (CHÁIDEZ, 2009). Medidas volumétricas permitem o cálculo do valor monetário dos bens e serviços que as florestas prestam à sociedade (ADEKUNLE et al., 2013). Para fins de manejo florestal e planejamento é vital saber o volume dos recursos de madeira e suas taxas de crescimento (ALTRIELL et al., 2010; THOMAS et al., 2006).

Essas estimativas podem ser mais confiáveis e robustas. Isso depende do desenvolvimento de equações de volume. Pela legislação florestal, as estimativas de volume para o primeiro Plano Operacional Anual (POA) podem ser calculadas por qualquer equação. No entanto, isso pode resultar em sub ou superestimativas. Por outro lado, em um segundo ponto há obrigatoriedade do desenvolvimento de uma equação específica (AGUIAR et al., 2017). Apresentar equações volumétricas consistentes e robustas são importantes para uso no manejo florestal. Isso diminuirá desperdício, evitará discrepâncias entre o volume estimado na floresta e o processado.

Com isso, para o licenciamento a partir da segunda Unidade de Produção Anual o órgão ambiental somente expedirá autorização de exploração mediante os resultados do inventário pré-exploratório com base em cálculos de volume a partir de equações específicas ao sítio florestal objeto do Plano de Manejo Florestal Sustentável (PMFS), conforme descrito no Art. 10 da Resolução CONAMA 406/2009. Assim, este estudo teve como objetivo ajustar modelos matemáticos e selecionar o com melhor critério para determinação de estimativa volumétrica para todas as espécies comerciais acima de $50 \mathrm{~cm}$ de diâmetro a altura do padrão (DAP), para uso exclusivo no planejamento florestal da área de manejo no município de Placa, estado do Pará, Brasil. 


\section{MATERIAIS E MÉTODOS}

A área de estudo está localizada no município de Placas-PA de coordenadas 54³4'30,00"W; $03^{\circ} 31^{\prime} 30,00^{\prime \prime} \mathrm{S}$, pertencente a Mesorregião do Baixo Amazonas (Figura 1). O clima da região é do tipo Afi, de acordo com a classificação climática de Köppen, caracterizado como tropical úmido, sem estação fria, com temperatura mínima média anual de $16^{\circ} \mathrm{C}$ e máxima média anual de $34^{\circ} \mathrm{C}$, com umidade relativa média de 91\%. A precipitação é do tipo convectiva, em forma de pancadas de curta duração. Apresenta valores anuais oscilando em torno de $2.000 \mathrm{~mm}$ e o trimestre mais seco ocorre de setembro a novembro; o trimestre mais chuvoso vai de fevereiro a abril. O solo é ácido, profundo e de atividade físico-química muito baixa, constituído por material mineral, do tipo Latossolo Amarelo distrófico (FUNDAC, 2005).

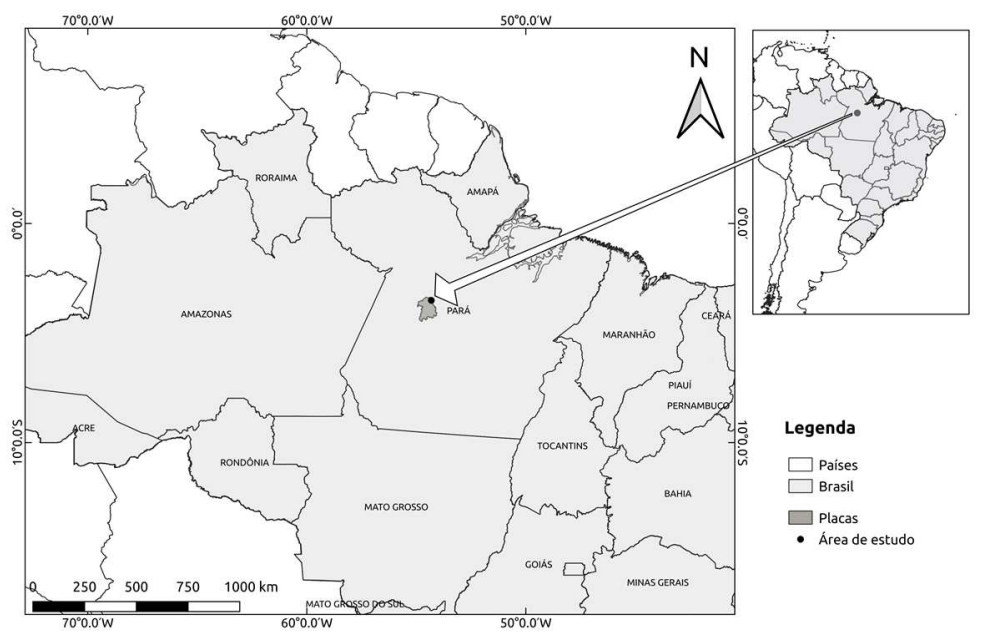

Figura 1: Localização da área de manejo florestal no município de Placas-PA.

A vegetação é do tipo floresta ombrófila densa de terra firme, seguindo a terminologia proposta (VELOSO et al., 1991). Nesse tipo de vegetação, ocorrem árvores de grande porte, trepadeiras lenhosas e epífitas em abundância, podendo ocorrer período seco de até 60 dias por ano (IBGE, 1992).

\section{Amostragem e coleta de dados}

A amostra foi composta por 235 árvores, distribuídas entre 14 das 17 espécies de valor comercial autorizadas para corte na Unidade de Produção Anual (UPA). Estas árvores foram selecionadas a partir de uma amostragem aleatória simples (AAS), com todo rigor, para que todas as classes diamétricas a partir de $50 \mathrm{~cm}$ e com amplitude de $10 \mathrm{~cm}$ fossem representadas (Tabela 1). O método utilizado para o cálculo do volume, das toras com casca, foi o geométrico utilizando a fórmula de Smalian, como recomendado pelo Anexo I da Resolução CONAMA (2009), como pode ser observado na fórmula abaixo.

$$
V_{\text {tora }}=0,7854 *\left(\frac{D_{b}+D_{t}}{2}\right)^{2} * L
$$

Vtora $=$ Volume da tora com casca em $\mathrm{m}^{3}$; $\mathrm{Db}=$ Diâmetro da base $(\mathrm{m})$, obtido da média das duas medidas de diâmetros maior e menor (Cruz); $\mathrm{Dt}=$ Diâmetro do topo $(\mathrm{m})$, obtido da média das duas medidas de diâmetros maior e menor (Cruz); $\mathrm{L}=$ Comprimento da tora.

As árvores amostradas se distribuem entre todas as classes de diâmetro exploradas comercialmente. 
A tabela 1 tem a distribuição e a representatividade de cada classe para a amostra geral.

Tabela 1: Distribuição das 235 árvores de 14 espécies, por classe de diâmetro.

\begin{tabular}{lll}
\hline Classe de DAP $(\mathbf{c m})$ & No de árvores & (\%) \\
\hline $\mathbf{5 0}-\mathbf{6 0}$ & 49 & 20,85 \\
$\mathbf{6 0}-\mathbf{7 0}$ & 88 & 37,45 \\
$\mathbf{7 0}-\mathbf{8 0}$ & 29 & 12,34 \\
$\mathbf{8 0}-\mathbf{9 0}$ & 14 & 5,96 \\
$\mathbf{9 0}-\mathbf{1 0 0}$ & 26 & 11,06 \\
$\mathbf{1 0 0}-\mathbf{1 1 0}$ & 12 & 5,11 \\
$\mathbf{1 1 0}-\mathbf{1 2 0}$ & 5 & 2,13 \\
$\geq 120$ & 12 & 5,11 \\
\hline Total & 235 & 100,00 \\
\hline
\end{tabular}

\section{Modelos Testados}

Para o cálculo do volume de madeira foram utilizados os modelos estatísticos tradicionais, (CAMPOS et al., 2009) os quais estão listados na tabela 2.

Tabela 2: Modelos testados para estimar o volume comercial por meio do DAP e Hc.

\begin{tabular}{lll}
\hline No & Modelos & Autor \\
\hline $\mathbf{1}$ & $\mathrm{V}_{\mathrm{i}}=\beta 0+\beta 1^{*} \mathrm{DAPi}{ }^{2}+\varepsilon$ & Kopesky e Gehrhardt \\
\hline $\mathbf{2}$ & $\mathrm{V}_{\mathrm{i}}=\beta 0^{*} \mathrm{DAPi}{ }^{\beta 1}+\varepsilon$ & Husch \\
\hline $\mathbf{3}$ & $\mathrm{V}_{\mathrm{i}}=\beta 0+\beta 1^{*} \mathrm{DAPi}+\beta 2 * \mathrm{DAPi}^{2}+\varepsilon$ & Hohenadl e Kreen \\
\hline $\mathbf{4}$ & $\mathrm{V}_{\mathrm{i}}=\beta 0^{*}\left(\mathrm{DAPi}{ }^{2} \mathrm{Hci}\right)^{\beta 1}+\varepsilon$ & Spurr \\
\hline $\mathbf{5}$ & $\mathrm{V}_{\mathrm{i}}=\beta 0+\beta 1^{*}\left(\mathrm{DAPi}{ }^{*} \mathrm{Hci}\right)+\varepsilon$ & Spurr \\
\hline $\mathbf{6}$ & $\mathrm{V}_{\mathrm{i}}=\beta 0^{*} \mathrm{DAPi}{ }^{\beta 1} \mathrm{Hci}^{\beta 2}+\varepsilon$ & Schumacher e Hall \\
\hline
\end{tabular}

Onde: $\mathrm{V}_{\mathrm{i}}$ é o volume da i-ésima árvore em $\mathrm{m}^{3}$; DAPi é o diâmetro da i-ésima árvore a 1,3 metros do solo em $\mathrm{cm}$; Hc i é a altura comercial da i-ésima árvore em metros e $\varepsilon$ é o erro aleatório.

\section{Critérios para seleção de modelos}

Os modelos volumétricos testados foram 3 de simples entrada (DAP) como variável independente e 3 modelos de dupla entrada (3 com DAP e Hc). Foram calculados, coeficiente de correlação ajustado do modelo ( $R^{2}$ ajust), erro padrão da estimativa $\left(S_{y x}\right)$, a incerteza em percentual (Incerteza\%), a análise gráfica da distribuição dos resíduos (R\%), a significância dos coeficientes de cada modelo (Valor-p) e Critério de informação Akaike (CIA) como critério de seleção de modelos.

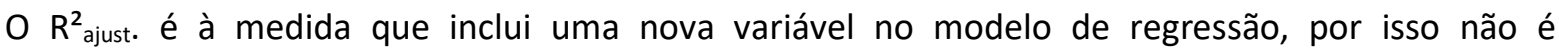
recomendado utilizá-lo isoladamente como critério de seleção. Devido a isso é comum utilizar o coeficiente de determinação ajustado para o número de coeficientes das equações (FIELD, 2009; SCHNEIDER et al., 2009).

(modelo)

$$
R_{\text {ajust. }}^{2}=\mathrm{R}^{2}\left[\frac{K-1}{N-K}\right] \cdot\left(1-R^{2}\right)
$$

Onde: $\mathrm{K}$ é o número de variáveis independente do modelo, $\mathrm{N}$ o número de observações, $\mathrm{R}^{2}$ o coeficiente de determinação.

O erro padrão da estimativa $\left(S_{y x}\right)$ e a $S_{y x}(\%)$ foram utilizados como critério para avaliação da qualidade dos modelos testados, prevalecendo aqueles que possuem os menores valores dessas estatísticas. As expressões para obtenção do $S_{y x}$ e a Incerteza, de acordo com o IPCC (2006) são:

(modelo )

$$
S_{\mathrm{yx}}=\sqrt{\frac{\sum_{\mathrm{i}=1}^{n}\left(V_{i}(\mathrm{obs})-V_{i}(\mathrm{est})\right)^{2}}{n-k}}
$$

Onde: $V_{i}$ (obs) é o volume observado da i-ésima árvore em $\mathrm{m}^{3} ; \mathrm{V}_{\mathrm{i}}$ (est) é o volume estimado da i-ésima árvore em $\mathrm{m}^{3}$; 
n é o número total de árvores amostradas e k é o número de coeficientes.

(modelo)

$$
\operatorname{Incerteza}(\%)=\left(\frac{z *\left(\frac{S_{\mathrm{yx}}}{\sqrt{n}}\right)}{\bar{V}_{(\mathrm{obs})}}\right) * 100
$$

Onde: Syx é o erro padrão da estimativa; z é a constante quando considerado um nível de confiabilidade de $95 \%$; $n$ é o número de árvores amostrada $\mathrm{e} \bar{V}_{\text {obs }}$ é a média aritmética do volume observado de todas as árvores amostradas. (modelo)

$$
R(\%)=\frac{\left(V_{i(\mathrm{est})}-V_{i(\mathrm{obs})}\right)}{V_{i(\mathrm{obs})}} * 100
$$

Onde: $V_{i(\text { est) }}$ é o volume estimado da i-ésima árvore em $\mathrm{m}^{3}$ e $\mathrm{V}_{\mathrm{i}(\mathrm{obs})}$ é o volume observado da i-ésima árvore em $\mathrm{m}^{3}$.

O ClA é uma forma simples, efetiva e objetiva para a seleção do melhor modelo aproximado (estimado), sendo um paradigma relativamente novo nas ciências estatísticas e biológicas já que difere em grande medida dos métodos usuais baseados em testes de hipóteses nulas (BURNHAM et al., 2010). O CIA pode ser calculado para cada possível combinação de variáveis explicativas, no qual o modelo com a menor CIA é escolhido como o modelo ótimo (ZUUR et al., 2007).

Na função genérica cálculo CIA é um estimador tendencioso do "log verossimilhança" e o viés assintótico é igual a K, o número de parâmetros livres no modelo (ANDERSON et al., 1994):

(modelo)

$$
\mathrm{CIA}=2 k-2 \log (L(ð / y))
$$

Em que K é o número de parâmetros estimadas (graus de liberdade) e $\mathcal{L}($ đ/y) é o log-verossimilhança no seu máximo ponto de o modelo estimado. O "2"é a constante (SNIPES et al., 2014).

Foi utilizado o cálculo do fator de forma para as 235 árvores cubadas na área de manejo florestal (BATISTA et al., 2014).

(Modelo)

$$
f=\frac{\sum V_{(i) \text { smalian }}}{\sum V_{(i) \text { cilindro }}}
$$

Onde: $f$ é o fator de forma, $\Sigma \mathrm{v}_{(\mathrm{i}) \text { smalian }}$ somatório do volume observado da i-ésima árvore obtido pela fórmula de

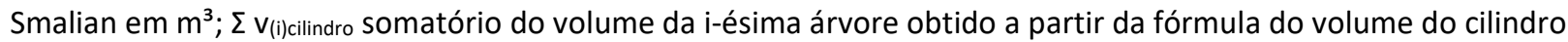
$\left(\left(\pi . \mathrm{DAP}^{2} / 40.000\right) * \mathrm{Hc}\right)$ em $\mathrm{m}^{3}$.

\section{Análise de dados}

Avaliação dos modelos para regressão foi feita com o software R studio versão 3.6.3. (Copyright (C) 2020 The R Foundation for Statistical Computing) para execução dos cálculos estatísticos (correlação Pearson a 95\%). Para tabulação e organização dos dados foi utilizado o sistema de planilha Libre Office Calc Versão: 6.4.0.3. Após o ajuste dos modelos foi realizada o teste-t de Student (Pareado) a 95\% de confiança, para verificar se existe diferença entre o volume cubado e volume estimado pelo modelo.

\section{RESULTADOS}

Para as 235 árvores cubadas obteve-se o fator de forma de 0,72, para as amostras da área de manejo florestal. Na tabela 3 está o número de árvores e a soma do volume por espécies (Smalian). A maior representação florística garante que a equação esteja condizente com as espécies a serem manejada no segundo ciclo de corte. Por isso, as espécies inventariadas devem estar contempladas em grande parte da amostragem destinada a equação de volume. 
Tabela 3: Volume comercial (soma) calculado pelo método de Smalian (Vol $\left(\mathrm{m}^{3}\right)$ ), média de DAP e média de altura comercial (Hc) para 235 árvores de 14 espécies.

\begin{tabular}{|c|c|c|}
\hline Nome científico & № árvores & $\operatorname{Vol}\left(\mathrm{m}^{3}\right)$ \\
\hline Apuleia molaris Spruce ex Benth. & 1 & 14,93 \\
\hline Astronium lecointei Ducke & 8 & 61,96 \\
\hline Caryocar villosum (Aubl.) Pers. & 14 & 322,58 \\
\hline Chrysophylum venezuelanense (Pierre) T.D.Penn. & 2 & 8,41 \\
\hline Dipteryx polyphylla Huber & 1 & 14,80 \\
\hline Enterolobium shomburgkii (Benth.) Benth. & 5 & 32,20 \\
\hline Goupia glabra Aubl. & 1 & 15,03 \\
\hline Handroanthus serratifolius & 2 & 9,50 \\
\hline Hymenaea courbaril L. var. courbaril & 31 & 288,17 \\
\hline Hymenolobium petraeum Ducke & 6 & 52,63 \\
\hline Manilkara huberi (Ducke) Chevalier & 150 & 854,15 \\
\hline Mezilaurus itauba (Meisn.) Taub. Ex Mez & 12 & 81,85 \\
\hline Microphocis venulosa (Mart. \& Eichler) Pierre & 1 & 3,96 \\
\hline Piptadenia suaveolens Miq. & 1 & 3,92 \\
\hline Total & 235 & 1764,09 \\
\hline
\end{tabular}

\section{Ajuste de equações}

Com o resultado dos volumes cubados para as espécies selecionadas realizou-se a análise dos modelos. Para esse ajuste as equações 1, 3 e 5 foram as mais precisas ao avaliar o CIA. As seis equações obtiveram um bom $\mathrm{R}^{2}{ }_{\text {ajust, }}$ Incerteza (\%) abaixo de $10 \%$ (Tabela 4), e boa dispersão de resíduos. Todas as equações foram submetidas ao teste-t de Student com valor de p a 95\% de confiabilidade. Com isso, foram obtidos resultados satisfatórios para análise, onde a média de volume de madeira não teve diferença do volume cubado. Assim, as estimativas das equações podem ser consideradas iguais ao mensurado pelo método de Smalian.

Tabela 4: Modelos testados (№), coeficientes estimados e suas respectivas estatísticas: Valor-p, Coeficiente de Determinação Ajustado ( $R^{2}$ ajust), Erro Padrão da Estimativa ( $\left.\mathrm{S}_{\mathrm{yx}}\right)$ em $\mathrm{m}^{3}$ e Incerteza em porcentagem (In \%), Critério de informação Akaike (CIA), volume estimado (VE $\left.\mathrm{m}^{3}\right)$, valor de $\mathrm{p}$ do test-t.

\begin{tabular}{|c|c|c|c|c|c|c|c|c|c|}
\hline \multirow{2}{*}{ Modelo } & \multicolumn{3}{|c|}{ Coeficientes (Valor-p) } & \multirow{2}{*}{$\mathbf{R}^{\mathbf{2}}$ adj } & \multirow[t]{2}{*}{$S_{y x}$} & \multirow[t]{2}{*}{$\ln \%$} & \multirow[t]{2}{*}{ CIA } & \multirow[t]{2}{*}{$\operatorname{VE}\left(m^{3}\right)$} & \multirow{2}{*}{ test-t (p) } \\
\hline & $\mathbf{a}$ & b & c & & & & & & \\
\hline 1 & 0,10486 & 11,84641 & 0,00000 & 0,938 & 0,088 & 2,29 & 140,96 & 1764,09 & 1,00 \\
\hline 2 & 11,98209 & 1,97531 & 0,00000 & 0,938 & 0,088 & 2,29 & 809,58 & 1765,70 & 0,98 \\
\hline 3 & $-0,46737$ & 1,28750 & 11,20071 & 0,938 & 0,088 & 2,29 & 142,62 & 1764,09 & 1,00 \\
\hline 4 & 0,46976 & 1,06307 & 0,00000 & 0,939 & 0,087 & 2,27 & 806,20 & 1752,00 & 0,92 \\
\hline 5 & $-0,43830$ & 0,59613 & 0,00000 & 0,937 & 0,088 & 2,30 & 145,36 & 1764,09 & 1,00 \\
\hline 6 & 2,19729 & 2,06194 & 0,55714 & 0,942 & 0,085 & 2,22 & 795,55 & 1757,95 & 0,96 \\
\hline
\end{tabular}

A dispersão de resíduos nos modelos de dupla e simples entrada obtiveram valores coesos (Figura 2). Na avaliação geral, todos tiveram boas dispersões ao se considerar o número de amostras utilizadas no ajuste. A análise da dispersão dos resíduos auxilia na seleção da melhor equação, devido ser possível visualizar se está ocorrendo sub ou superestimativa em alguma classe de tamanho. Por isso, a sua avaliação é fundamental para evitar a seleção de alguma equação tendenciosa.

$\mathrm{Na}$ avaliação do $\mathrm{ClA}$ o modelo 1 foi o com melhor resultado para o ajuste de equação volume para área de manejo florestal. A precisão do mesmo deve estar associada a alta correlação $(r=0,95)$ do DAP com o volume. A equação 5 foi a mais eficiente entre as de dupla entrada, onde a Hc teve correlação ruim $(r=-$ 0,23). Contudo, a utilização da altura nessas equações garantiu uma melhor dispersão de resíduos para as classes iniciais. Isso mostra que a altura influenciou positivamente na estimativa para essas classes quando 
comparadas as equações de simples entrada. Por isso, as melhores dispersões foram nessas equações.
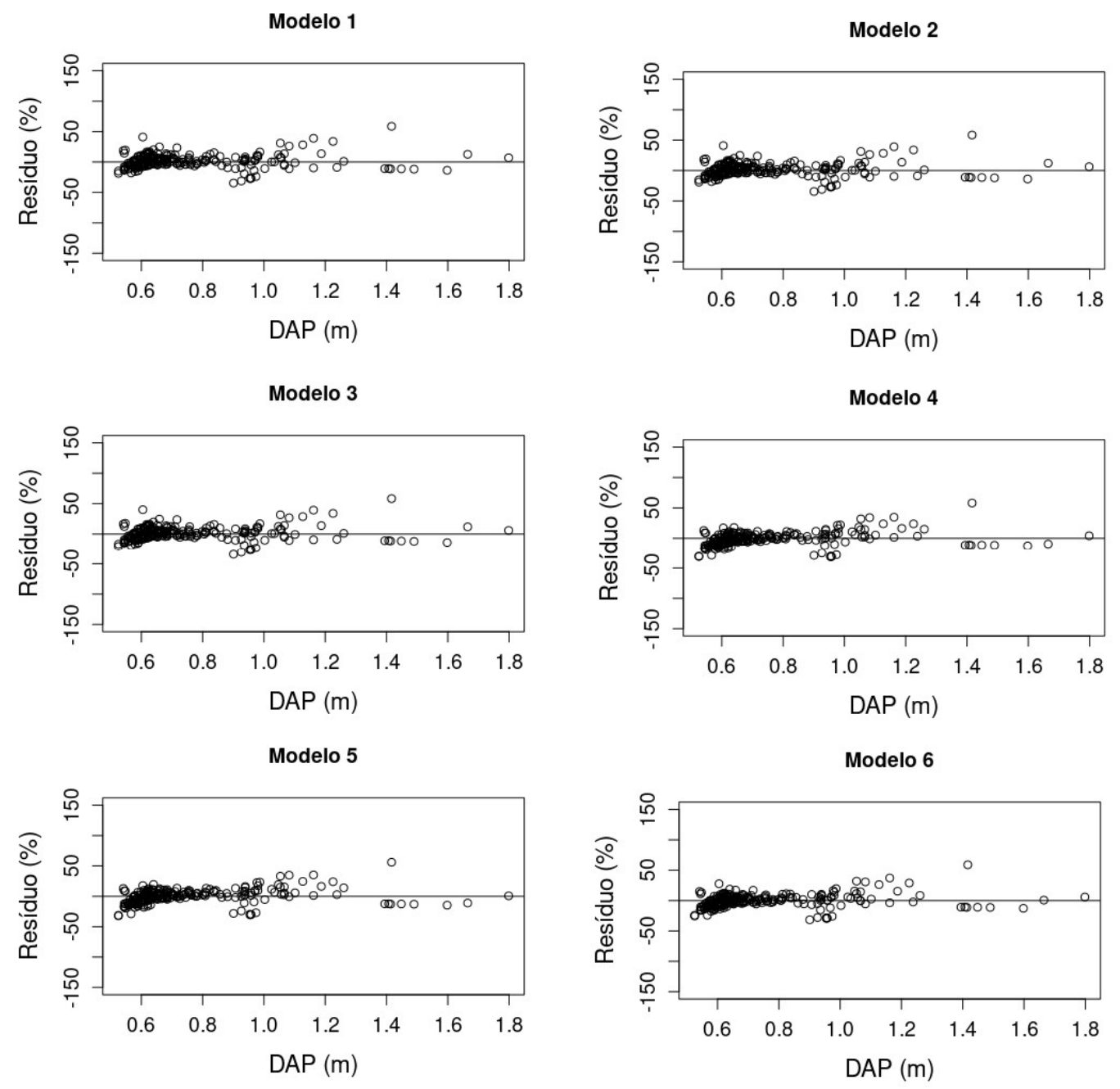

Figura 2: Gráficos de dispersão de resíduos para os modelos ajustado a partir da cubagem.

\section{DISCUSSÃO}

O fator de forma utilizado para espécies comerciais é de 0,70. Para outras áreas na floresta amazônica foram encontrados valores de 0,73 no estado de Roraima (GIMENEZ et al., 2015) e 0,74 no Mato Grosso (COLPINI et al., 2009). É importante saber a forma do fuste, pois pode causar diferenças significativas na determinação do volume explorado nas florestas tropicais do bioma Amazônico. Assim como o fator de forma, a correlação das variáveis independentes é imprescindível para estimativas volumétricas em planos de manejo florestal.

A medição de $\mathrm{Hc}$ das árvores em florestas tropicais é um desafio para as estimativas volumétricas por ser de difícil medição. É uma variável que quando obtida com boa precisão pode gerar equações mais eficazes para estimativas de volume, contudo, se não tiver boa correlação poderá ser inócua. Por isso, deve se ter cautela ao utilizar as variáveis independentes e considerar a correlação antes de testar os modelos.

Nos trópicos, os inventários florestais normalmente incluem o DAP das árvores e Hc. Em muitos casos, a Hc é difícil de se medir com segurança. Este problema resulta em estimativas tendenciosas quando a altura da árvore é incluída como uma variável independente em modelos de volume. Considerando-se essas 
fontes de erro, é necessário avaliar equações de volume que utiliza o DAP como variável independente. Esta pode ser medida com rigor e precisão no campo exige menos trabalho, e por isso é eficiente na redução de custo nos inventários florestais (SEGURA et al., 2005).

Equações de volume são utilizadas para estimativas volumétricas de árvores de vários tamanhos e espécies. A confiabilidade das estimativas de volume depende do alcance e extensão da amostra de dados disponíveis e como equações de volume podem encaixar esses dados amostrais (AVERY et al., 2002). O volume total do estoque de floresta em crescimento é uma variável requerida no manejo florestal. Normalmente, o volume é calculado como o volume total por unidade de área, enquanto são utilizados modelos prevendo o volume total das árvores (MASOTA et al., 2014). Com isso, estimativas errôneas do volume de madeira podem acarretar prejuízos financeiros, assim como, problemas no planejamento do manejo futuro.

Esse estudo teve resultados que se diferenciaram dos trabalhos de Barros et al. (2009), Silva et al. (1984), Thaines et al. (2010) e Tonini et al. (2015) nos quais os modelos com a Hc obtiveram melhores coeficientes. Problemas como a falta de perícia nas estimativas ou mesmo erros de medição podem estar associados a isso. Assim, recomenda-se o uso de metodologias que garantam maior precisão na coleta de variáveis e que sejam aplicadas sem comprometer a produtividade, tanto do inventário florestal quanto do manejo florestal.

\section{CONCLUSÕES}

As avaliações das 6 equações tiveram resultados satisfatórios, sendo que com os parâmetros analisados qualquer uma poderia ser utilizada na estimativa volumétrica para o segundo POA. Isso, porque ambas obtiverem baixo erro de estimativa e bons $R^{2}$ ajust, boa dispersão de resíduos, além de volumes estimados equivalente ao da cubagem. Diante da grande incerteza quanto à obtenção da Hc as equações de simples entradas foram tão eficientes quanto as de dupla entrada para estimativas de volume de madeira desse plano de manejo. Assim, o ajuste das equações teve sua precisão sustentada pela alta correlação do DAP para as árvores amostradas.

AGRADECIMENTOS: À Associação dos Agricultores Rainha da Floresta Comunidade Sombra Santa por terem disponibilizado os dados para a publicação.

\section{REFERÊNCIAS}

ADEKUNLE, V. A. J.; NAIR, K. N.; SRIVASTAVA, A. K.; SINGH, N. $K$.. Models and form factors for stand volume estimation in natural forest ecosystems: a case study of Katarniaghat Wildlife Sanctuary (KGWS), Bahraich District, India. Journal of Forestry Research, v.24, n.2, p.217-226, 2013.

AGUIAR, D. R.; LIMA, A. J. N.; GAMA, J. R.; ANDRADE, D. F. C.; SANTOS, J.; HIGUCHI, N.. Adjustment of volumetric equations from fallen trees for analysis of the logging effect in the Tapajós National Forest, Pará, Brazil. Australian Journal of Basic and Applied Sciences, v.11, n.10, p.48-59,
2017

AKINDELE, S. O.; DYCK, J.; AKINDUNNI, F. F.; PAPKA, P. M.; OLALEYE, O. A.. Estimates of Nigeria's Timber Resources. In: POPOOLA, L.; ABU, J. E.; ONI, P. I.. Forestry and National Development. In: ANNUAL CONFERENCE OF THE FORESTRY ASSOCIATION OF NIGERIA, 27. Annals. Abuja, 2001. p.1-11.

ALTRIELL, D.; BRANTHOMME, A.; TAVANI R.. Assessing growing stock and stock changes through multi-purpose national forest monitoring and assessment. Rome: FAO 
Forest Resources Assessment Programme Working paper, 2010.

ANDERSON, D. R.; BURNHAM, K. P.; WHITE, G. C.. AIC model selection in overdispersed capture-recapture data. Ecology, v.75, p.1780-1793, 1994.

AVERY, T. E.; BURKHART, H. E.. Forest measurements. 5 ed. New York: McGraw-Hill Higher Education, 2002.

BARROS, P. L. C.; SILVA JÚNIOR, A. T.. Equação de volume para árvores de uma floresta tropical densa no município de Anapu, oeste do Estado do Pará, Amazônia Oriental. Revista de Ciências Agrárias, Belém, n.51, p.115-126, 2009.

BATISTA, J. L. F.; COUTO, H. T. Z.; SILVA FILHO, D. F.. Quantificação de recursos florestais: Árvore, arvoredos e florestas. São Paulo: Oficina de Textos, 2014.

BURNHAM, K. P.; ANDERSON, D. R.. Information and likelihood theory: a basis for model selection and inference. In: Model selection and multimodel inference. 2 ed. New York: Springer, 2010. p.49-97.

CAMPOS, J. C. C.; LEITE, H. G.. Mensuração florestal: perguntas e respostas. Viçosa: UFV, 2009.

CHÁIDEZ, J. N.. Allometric equations and expansion factors for tropical dry forest trees of Eastern Sinaloa, Mexico. Tropical and Subtropical Agro-ecosystems, v.10, p.45-52, 2009.

COLPINI, C.; TRAVAGIN, D. P.; SOARES, T. S.; SILVA, V. S. M.. Determinação do volume, do fator de forma e da porcentagem de casca de árvores individuais em uma Floresta Ombrófila Aberta na região noroeste de Mato Grosso. Acta Amazônica, v.39, n.1, p.97-104, 2009.

CONAMA. Resolução no.411, de 6 de maio de 2009. Dispõe sobre procedimentos para inspeção de indústrias consumidoras ou transformadoras de produtos e subprodutos florestais madeireiros de origem nativa, bem como os respectivos padrões de nomenclatura e coeficientes de rendimento volumétricos, inclusive carvão vegetal e resíduos de serraria. Brasília: DOU, 2009.

ETIGALE, E. B.; OLAJIDE, O.; UDO, E. S.. Stand structure, density and yield of tree community in Ukpon River Forest Reserve, Cross River State, Nigeria. Nature and Science, v.12, n.11, 2014.

FIELD, A.. Descobrindo a estatística usando SPSS. 2 ed. Porto Alegre: Artmed, 2009.

FUNDAC. Fundo de Desenvolvimento e Ação Comunitária. Plano de Desenvolvimento Sustentável Moju I e II. Santarém: FUNDAC, 2005.
IPCC. Intergovernmental Panel on Climate Change. IPCC Guidelines for National Greenhouse Gas Inventories. Prepared by the National Greenhouse Gas Inventories Programme. Tokyo: IGES, 2006.

GIMENEZ, B. O.; DANIELLI, F. E.; OLIVEIRA, C. K. A.; SANTOS, J.; HIGUCHI, N.. Equações volumétricas para espécies comerciais madeireiras do sul do estado de Roraima. Scientia Forestalis, Piracicaba, v.43, n.106, p.291-301, 2015.

IBGE. Instituto Brasileiro de Geografia e Estatística. Manual técnico de vegetação brasileira. Rio de Janeiro, IBGE, 1992.

MASOTA, A. M.; ZAHABU, E.; MALIMBWI, R. E.; BOLLANDSÅS, O. M.; EID, T. H.. Volume models for single trees in tropical rainforests in Tanzania. Journal of Energy and Natural Resources, v.3, n.5, p.66-76, 2014.

SEGURA, M.; KANNINEN, M.. Allometric models for tree volume and total aboveground biomass in a Tropical Humid Forest in Costa Rica. Biotropica, v.37, n.1, p.2-8, 2005

SCHNEIDER, P. R.; SCHNEIDER, P. S. P.; SOUZA, C. A. M.. Análise de regressão aplicada a engenharia florestal. 2 ed. Santa Maria, 2009.

SILVA, J. N. M.; ARAUJO, S. M.. Equação de volume para árvores de pequeno diâmetro na Floresta Nacional do Tapajós. Boletim de Pesquisa Florestal, Colombo, n.8-9, p.16-25, 1984

SNIPES, M.; TAYLORN, D. C.. Model selection and Akaike Information Criteria: An example from wine ratings and prices. Wine Economics and Policy, v.3, p.3-9, 2014.

THAINES, F.; BRAZ, E. M.; MATTOS, P. P.; THAINES, A. A. R.. Equações para a estimative de volume de madeira para a região da bacia do Rio Ituxi, Lábrea, AM. Pesquisa Florestal Brasileira, Colombo, v.30, n.64, p.283-289, 2010. DOI: http://doi.org/10.4336/2010.pfb.30.64.283

THOMAS, C.; ANDRADE, C. M.; SCHNEIDER, P. R.; FINGER, C. A. G.. Comparação de equações volumétricas ajustadas com dados de cubagem e análise de tronco. Ciência Florestal, Santa Maria, v.16, n.3, p.319-327, 2006.

TONINI, H.; BORGES, R. A.. Equação de volume para espécies comerciais em Floresta Ombrófila Densa no sul de Roraima. Pesquisa Florestal Brasileira, v. 5, p.111-117, 2015.

VELOSO, H.; RANGEL FILHO, A. L. R.; LIMA, J. C. A.. Classificação da vegetação brasileira, adaptada a um sistema universal. Rio de Janeiro: IBGE, 1991.

ZUUR, A. F.; IENO, E. N.; SMITH, G. M.. Analyzing ecological data. New York: Springer, 2007.

A CBPC - Companhia Brasileira de Produção Científica (CNPJ: 11.221.422/0001-03) detém os direitos materiais desta publicação. Os direitos referem-se à publicação do trabalho em qualquer parte do mundo, incluindo os direitos às renovações, expansões e disseminações da contribuição, bem como outros direitos subsidiários. Todos os trabalhos publicados eletronicamente poderão posteriormente ser publicados em coletâneas impressas sob coordenação da Sustenere Publishing, da Companhia Brasileira de Produção Científica e seus parceiros autorizados. Os (as) autores (as) preservam os direitos autorais, mas não têm permissão para a publicação da contribuição em outro meio, impresso ou digital, em português ou em tradução. 